\title{
Issue 2
}

\section{S. V. Raghavan}

Received: 3 June 2013/Accepted: 3 June 2013/Published online: 18 June 2013

(C) CSI Publications 2013

We are now having our second issue of CSI Transactions on ICT. This issue comes at a time when the world is gripped with the larger problem of securing the Cyber Space. Extensive discussions in various policy forums and professional conferences in last few years seem to point out that securing Cyber Space is not only a non-trivial task, but also an extremely challenging one. There is general acknowledgement of the fact that developing ones own hardware and software ecosystem along with a robust and dependable supply chain management is important to creating a secure Cyber Space. In this issue we have some articles focusing on different aspects of robust Cyber Space.

Darshana Hooda and Parvinder Singh look at parameterized security technique based on remote reference passing (RRP), which intrinsically works on the principle of transmitting information in the form of reference at the receiver side as the most suitable technique to address security needs of video services when accessed/played through constrained devices with low power design while at the same time ensuring negligible delay during decryption process.

Gandharba Swain and Saroj Kumar Lanka explore two sided, three sided and four sided steganography methods to take embedding decision, while keeping the fall off boundary problem (FOBP) and fall in error problem (FIEP) in focus. These methods possess large hiding capacity, better imperceptibility and high security.

Durga Samanth Pidikiti, Rajesh Kalluri and R K Senthil Kumar explore security of hierarchical SCADA systems

S. V. Raghavan $(\bowtie)$

Chennai, India

e-mail: sv.raghavan@inc.in that use communication protocols, such as IEC 608705-101 and IEC 60870-5-104 protocols (in power utilities sector) that seem to fall short of application layer and the data link layer security. Application layer security is necessary to protect the SCADA systems from spoofing and non-repudiation attacks. Data link layer security is necessary to protect the systems from the sniffing, data modification and replay attacks. Authors propose an experimental research model that can be used to mitigate the attacks at application layer and data link layer by adopting the IEC 62351 standards.

Kakali Chatterjee, Daya Gupta, and Asok De present an insight in to the design, build and deployment of secure systems by integrating security in the application development life cycle. They propose a Framework for Security Engineering Process that involves converting security requirements and threats into design decisions to mitigate the identified security threats. The identified design attributes are prioritized and a security design template is prepared.

Chabbi Rani Panigrahi and Rajib Mall propose a regression test case prioritization technique for object-oriented programs. They construct an intermediate graph model of a program from its source code. When the program is modified, the model is updated to reflect the changes. The model captures control and data dependencies, and information pertaining to various types of dependencies arising from object-relations such as association, inheritance and aggregation. Experimental results indicate that their approach achieves an increase in the APFD metric by $25.70 \%$ as compared to contemporary approaches.

Of course, high performance computing (HPC) is becoming ubiquitous. Energy concerns are deep and green technologies are evolving. 
Bhavyasree Unni, Nazia Parveen, and B S Bhindhumadava explore Energy-Aware Computing as it is gaining more and more attention in HPC environment. They propose a novel energy optimization approach for Message Passing Interface (MPI) applications running on HPC systems by applying dynamic voltage frequency scaling (DVFS) at node level using an optimization agent and running the CPU cores at reduced frequencies without compromising performance.

India is a linguistically rich country having eighteen constitutional languages, which are written in ten different scripts. Indian languages are highly inflectional with a rich morphology, relatively free word order, and default sentence structure as subject object verb. Many of them are structurally similar called sibling languages. Hindi and Gujarati languages are such siblings.

Kalyani A Patel and Jyothi S Pareek concentrate on language translation. They describe GH-MAP; a rule based token mapping system developed by us for translation between sibling language pair Hindi and Gujarati. GH-MAP system performs effective word-for-word translation using simple and computationally inexpensive methods and minimal lexical resources. Issues of syntactic, semantic and structural divergence in translation using GH-MAP are resolved with the help of special empirical rules. The aim of GH-MAP is not to produce high quality translation in the sense of linguistics; rather it has been developed to produce correct working translation just enough to cross the language barrier. The system was evaluated on the test bed obtained from FIRE 2010, literature on Gandhiji and ELRA-W0037. For establishing relevance of the model, 'into-Gujarati' BLEU, PER and METEOR score have been calculated. We see this as an important work indeed for India.

CSI Transactions editorial board has decided through electronic deliberations that subsequent issues will start focusing on specific themes. Soon you will see an announcement in our journal web site in this regard. On the whole, the Transactions is gaining strength and is set to scale greater heights in the coming months.

Happy reading. 